www.jmscr.igmpublication.org Impact Factor 5.244

Index Copernicus Value: 83.27 ISSN (e)-2347-176x ISSN (p) 2455-0450 crossref DOI:_http://dx.doi.org/10.18535/jmscr/v4i8.58

\title{
Diabetic Nephropathy: Ultrasound, Color Doppler and Biochemical Correlation- A 2 Year Study
}

\author{
Authors \\ Manish Shaw ${ }^{1}$, S.Bhagat ${ }^{2}$, B.B.Panda ${ }^{3}$, S. Nisa ${ }^{4}$, B.Das ${ }^{5}$, Amulya Panda ${ }^{6}$ \\ ${ }^{1,6}$ Post graduate Students, Department of Radio diagnosis, VIMSAR, Burla, Odisha, India, 768017 \\ ${ }^{2}$ Head and Professor, Department of Radio diagnosis, VIMSAR, Burla, Odisha, India \\ ${ }^{3,4,5}$ Associate Professor, Department of Radio diagnosis, VIMSAR, Burla, Odisha, India \\ Corresponding Author \\ Manish Shaw \\ Email: manish4u88@gmail.com, Contact: 9658421256
}

\begin{abstract}
Aims \& Objectives- To evaluate the biochemical parameters, sonomorphological and Doppler ultrasound findings in assessment of diabetic nephropathy and correlation among them.

Materials \& methods-60 patients having type II diabetes mellitus who were referred to our department from December 2013 to September 2015 for either routine abdominal ultrasound or renal Doppler are randomly selected, categorised (4 subgroups) and studied using Philips HD 7 USG machine. Both kidneys were studied for dimensions, parenchyma thickness, echogenicity and resistive indices from interlobar arteries and biochemical tests were analysed by collecting the investigation reports. Correlations were studied between sonomorphological, Doppler USG and biochemical parameters. Statistical significance was ascertained using Microsoft Excel v10 (with data analysis tool kit) \& Epi-Info $v 7$.

Results- All patients in preclinical and incipient subgroup; more than half of the patients with renal failure were found to have normal renal parenchymal echogenicity. Around one third and $18.2 \%$ of patients had grade I and grade II cortical echogenicity changes in renal failure subgroup respectively. Most of the patients in preclinical group had normal RI value while patient belonging to other 3 subgroups had raised RI value (>0.7) with highest values in Renal failure subgroup.

Conclusions- Renal sonomorphological changes were noted in few patients and only in advanced stages of diabetic nephropathy but Resistive indices values were increased in most of the patients belonging to subgroups 2,3 and 4 and even in patients having normal sonomorphological features. Resistive indices values showed positive correlation with serum creatinine and blood urea nitrogen values suggesting complementary role of Doppler ultrasound in Diabetic nephropathy.
\end{abstract}

Keywords: Diabetic Nephropathy, Renal Doppler, Resistive Index.

\section{Introduction}

In the modern era of evidence based medicine, Ultrasound \& Doppler studies are the most important investigation sought by the clinicians. The Duplex ultrasound is a reliable, non-invasive \& easily available method. There is no need for contrast agents as such and thus even in renal failure patients, it can be used effectively. According to the latest 2015 report 
of International Diabetes Federation (IDF) ${ }^{[1]}$, there are 69.2 million diabetic people living in India with a prevalence of $9.3 \%$ and affection at early age. About one third of diabetic patient suffer from nephropathy and it is an irreversible microvascular complication ${ }^{[2]}$. Platt ${ }^{[3]}$ has stated that Doppler study has the potential to provide physiological information about renal vascular resistance. Parolini et al [4] 2009, have showed that Resistive Indices (RI) measurements are useful to evaluate the extent of renal impairment \& long term renal prognosis in chronic nephropathies diseases like diabetic renal disease and hypertensive nephropathy. The complimentary role of Ultrasound and promising potential of Doppler study to assess the intrarenal vasculature in this large number of people suffering from diabetic renal disease were the motivating factor to do this study. A non-invasive imaging technique like Duplex ultrasound to detect subtle changes in the initial stages of Diabetic Nephropathy, suggesting the need for aggressive and accurate management would be highly desirable.

This study was conducted with the hypothesis that Duplex ultrasound has a role in detection and follow up of patients in initial stages of Diabetic Nephropathy(DN) as it can provide valuable information about the intra renal hemodynamic abnormalities like intrarenal arteriosclerosis and to investigate the correlation between biochemical and Duplex ultrasound parameters especially RI values.

\section{Aims \& Objectives}

1. Evaluation of sonomorphological characteristics and renal vascular resistance (Resistive Index) values using Duplex ultrasound in patients with diabetic renal disease.

2. Correlation between Duplex ultrasound and Biochemical parameters viz. Blood Urea Nitrogen \& Serum Creatinine.

\section{Materials And Methods}

The study was carried out in the Department of Radiodiagnosis, VSS Medical College and Hospital, Burla, by PHILIPS HD 7 USG machine (Fig1) from December 2013 to November 2015 with informed consent involving 60 patients with 46 males and 14 female patients.

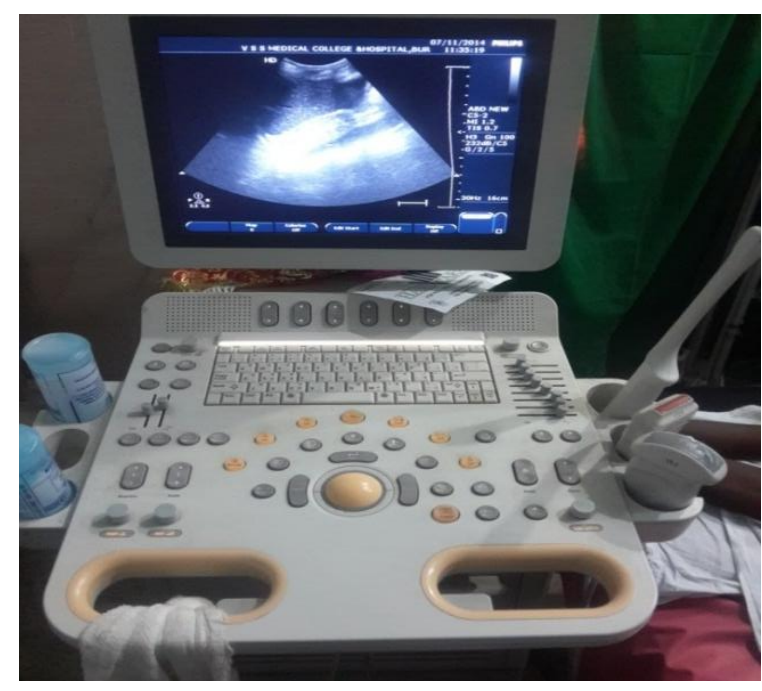

Fig1: Philips HD 7 USG machine in dept. of VIMSAR

This study was approved by our institutional ethical committee. It was an observational and Cross sectional study. Inclusion criteria were patients diagnosed as type $2 \mathrm{DM}$ and above 20 years. Patients having urinary tract infection, unilateral or bilateral contracted kidneys on Sonography, fatty liver or other liver disorders and cardiovascular diseases were excluded from the study. Biochemical parameters like Fasting Blood Sugar (FBS), Blood Urea Nitrogen (BUN) and Serum Creatinine were recorded in all patients. Urine analyses for urine protein were done by heat $\&$ acetic method and in selected cases for microalbuminuria were done by spot urine albumin/ creatinine ratio and noted as present or absent. Dissanayake et al ${ }^{[5]}$ highlighted that proteinuria of $>0.5 \mathrm{gm} / 24$ hours can be detected by standard heat \& acetic acid method (1+ or more). 24 hour urinary excretion rates provides the most precise 
measurement (30-300 mg/24 hours) but alternatively it can be diagnosed in office setting by spot urine Albumin/ Creatinine Ratio (ACR) of 3-30 mg/millimol ${ }^{[6] \text {. }}$

Patients were divided into 4 subgroups. SUBGROUP1: PRECLINICAL- Type 2 DM patients without any clinical/ biochemical indicators of DN $(n=17)$. SUBGROUP2: INCIPIENT NEPHROPATHY- Type 2 DM patients with presence of microalbuminuria (ACR of $3-30 \mathrm{mg} / \mathrm{millimol}$ ) which remains undetected by routine urine protein analysis methods $(n=11)$. SUBGROUP3: OVERT NEPHROPATHY- Type 2 DM patients with macroproteinuria, detected by routine urine protein analysis but without any features of renal failure like raised serum creatinine $(n=10)$. SUBGROUP4: RENAL FAILUREType $2 \mathrm{DM}$ patients with features of renal failure reflected by increased serum creatinine $(\geq 1.5 \mathrm{mg} / \mathrm{dl})(\mathrm{n}=22)$.

Both kidneys were evaluated by Philips HD-7 Colour Doppler ultrasound machine using a convex array probe (C5-2) having 2-5 MHz extended operator frequency range with default abdominal setting was used to evaluate general ultrasound parameter and renal vascular setting for Duplex ultrasound study of renal vessels. B-MODE conventional ultrasound parameters measured were the length of the kidneys, parenchymal thickness (PT) and renal parenchymal (cortical) echogenicity. The echogenicity was graded as

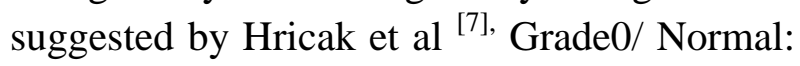
The echogenicity of renal cortex is less than that of liver (Fig2); Grade 1: The echogenicity of renal cortex is equal to that of liver (Fig3); Grade 2: The echogenicity of renal cortex is more than that of liver but less than renal sinus (Fig4) and Grade 3: The echogenicity of renal cortex is equal to renal sinus. Patients having Grade 3 disease were not included as it is not possible to measure the exact parenchymal thickness in these patients. ${ }^{[8]}$

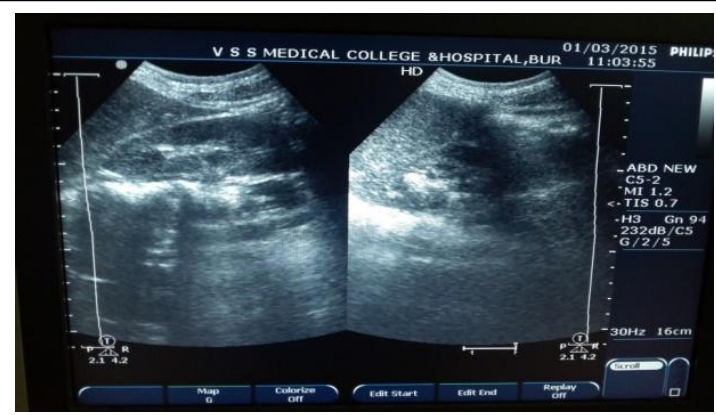

Fig 2: B/L Kidney showing normal (grade 0) parenchymal echogenicity.

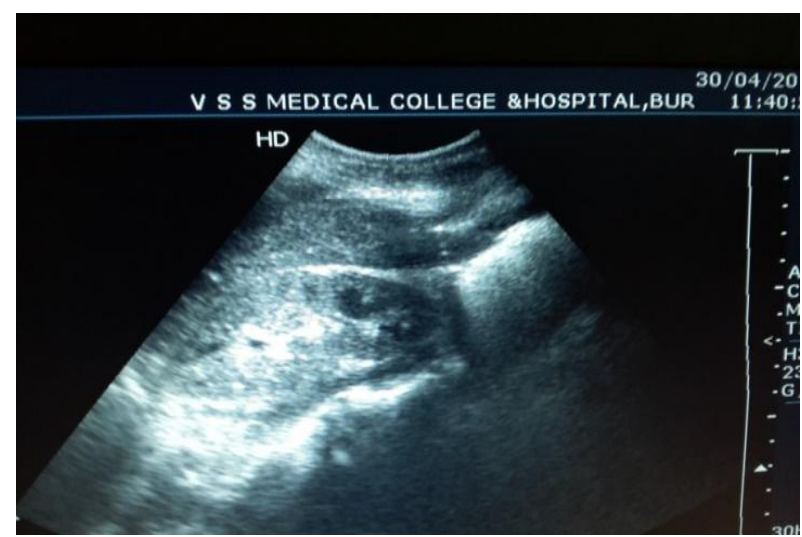

Fig 3: Right kidney showing grade 1 parenchymal echogenicity

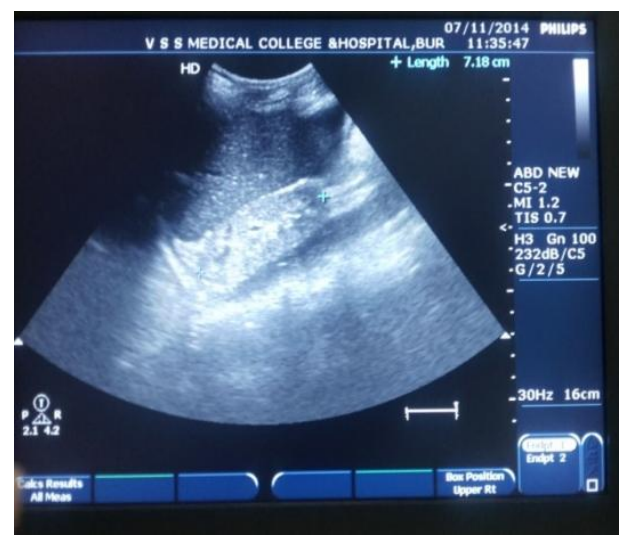

Fig 4: Left kidney showing grade 2 parenchymal echogenicity

In all patients, Pulsed Doppler measurements (superimposed on Color Doppler \& B mode ultrasound image) in interlobar arteries were performed on both kidneys at perimedullary area in at least 3 distinct vessels in each kidney and measurements were only taken when at least 3 consecutive similar waveforms were observed (Fig.5 \& 6). 
Flow chart of renal artery:

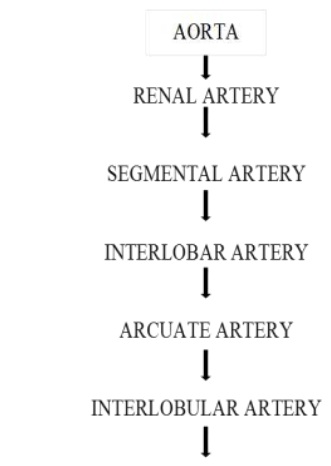

AFFERENT ARTERIOLE AND GLOMERULUS

Fig 5. Renal artery anatomical sequence

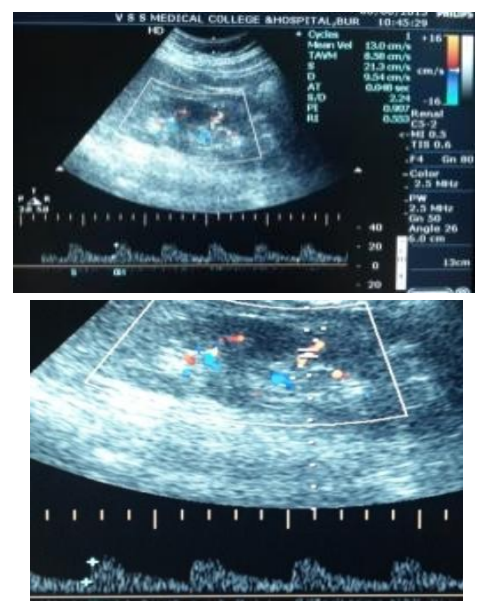

Fig 6: Procedure for measurement of RI in Interlobar artery.

Doppler sample volume was set at 2-4 $\mathrm{mm}$ \& angle was adjusted to less than 60 degrees. The lowest possible wall filter $(50 \mathrm{~Hz})$ was used. Average time for each patient was about 30 minutes. According to Pourcelot, ${ }^{[9]}$ the resistive index is measured as: $\mathrm{RI}=1$ $E D V / P S V$, and the same was calculated by the automated system in ultrasound machine (Fig $7,8 \& 9)$.

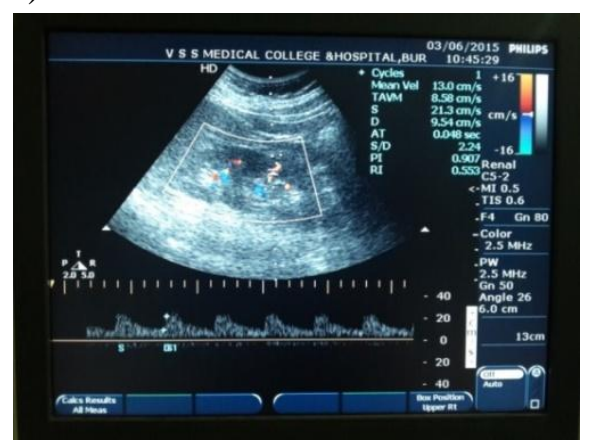

Fig.7: Renal Doppler Indices in one of the patient belonging to preclinical subgroup

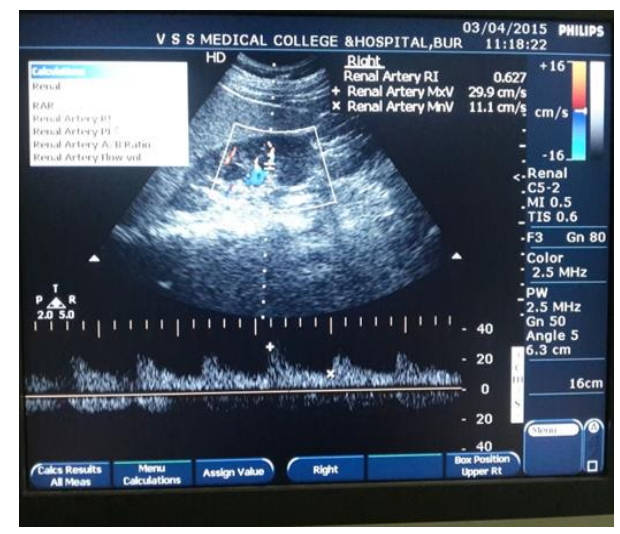

Fig 8: Right Renal Interlobar Artery RI in a patient of Incipient Nephropathy

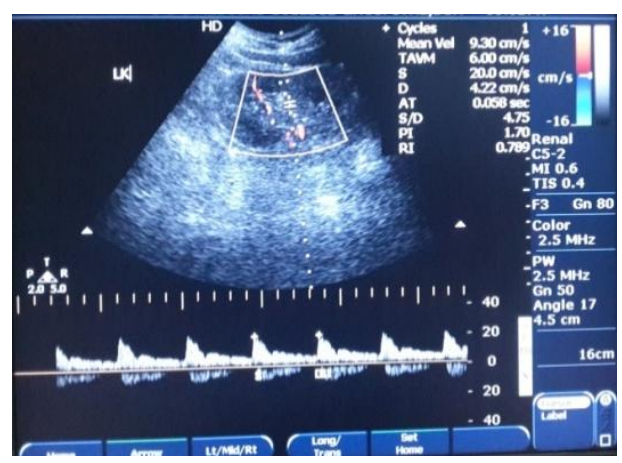

Fig 9: High RI measured in a renal failure diabetic patient

RI values from upper, mid \& lower poles of each kidney were obtained and mean of the six values thus obtained was calculated. An RI value of more 0.70 was considered abnormal [10].

One way Anova test was used to calculate the significance between differences in more than 2 means and Pearson's correlation (with linear regression analysis) was drawn between biochemical, ultrasound \& Doppler parameters by statistical analysis using Microsoft Excel v10 (with data analysis tool kit) \& Epi-Info v7. $P$ value $<0.05$ was taken as significant.

\section{Results}

60 patients diagnosed with type 2 DM underwent general ultrasound and Duplex evaluation of both kidneys. Biochemical parameters like FBS, BUN, Serum Creatinine $\&$ urine protein was recorded in all patients. 28 patients having negative urine protein and normal serum creatinine underwent spot urine 
examination for detection of microalbuminuria. There were 46 male patients constituting $76.67 \%$ of study group and 14 female patients constituting $23.33 \%$ of the study group.

The mean duration of Diabetes Mellitus (DM) (in years) in preclinical, incipient nephropathy, overt nephropathy and renal failure subgroup were 6.88 \pm 3.98 Standard Deviation (SD), 8.27 $\pm 4.51 \mathrm{SD}, \quad 10.8 \pm 4.51 \mathrm{SD}, \quad 11.68 \pm 5.65 \mathrm{SD}$ respectively. With increase in the mean duration of DM, there was also progressive increase in DN severity, which was found to be statistically significant $(P=0.02)$.

In this study, the mean renal length was highest in preclinical subgroup but no steady relationship can be deduced with the severity of DN. In this study, the smallest kidneys were noted in patients having DM for more than 15 years but the variation in renal length with duration of disease does not follow a linear relationship. The mean renal length of left kidney was found to be greater than right kidney in almost all subgroups depending either on severity or on duration of disease.

The mean renal parenchymal thickness $\{$ in millimetre $(\mathrm{mm})\}$ of right kidney in preclinical, incipient nephropathy, overt nephropathy and renal failure subgroup were 15.52 $\pm 2.08 \mathrm{SD}, 15.45 \pm 2.53 \mathrm{SD}, 15.00 \pm 1.34 \mathrm{SD}$, $14.59 \pm 2.34 \mathrm{SD}$ respectively. With increasing severity of DN, the parenchymal thickness was noted to decrease but it was not statistically significant $(P=0.57)$.

The mean renal parenchymal thickness (in $\mathrm{mm}$ ) of left kidney in preclinical, incipient nephropathy, overt nephropathy and renal failure subgroup were 16.88 $\pm 2.88 \mathrm{SD}$, $16.00 \pm 3.10 \mathrm{SD}, 15.30 \pm 2.00 \mathrm{SD}, 15.27 \pm 3.40 \mathrm{SD}$ respectively. With increasing severity of $\mathrm{DN}$, the parenchymal thickness was noted to decrease but it was not statistically significant $(P=0.40)$.

All the patients in preclinical and incipient nephropathy subgroup had normal renal parenchymal echogenicity. $70 \%$ of overt nephropathy patients had normal echogenicity while $20 \%$ \& $10 \%$ of patients in this subgroup had Grade 1 \& 2 parenchymal echogenic changes. In renal failure subgroup, $50 \%$ of patient had normal echogenicity while $31.8 \%$ $\& 18.2 \%$ of patients showed Grade $1 \& 2$ renal parenchymal echogenic changes.

The mean RI values in preclinical, incipient nephropathy, overt nephropathy and renal failure subgroup were $0.641 \pm 0.06 \mathrm{SD}$, $0.724 \pm 0.10 \mathrm{SD}, 0.756 \pm 0.05 \mathrm{SD}, 0.820 \pm 0.09 \mathrm{SD}$ respectively, showing progressive increase in mean RI values with increase in severity of DN, which was found to be statistically significant $(P<0.001)$. In preclinical stage, the mean RI value of $0.641 \pm 0.06 \mathrm{SD}$ is in normal range $(<0.70)$. However, in presence of microalbuminuria, the mean RI values are higher $(0.724 \pm 0.10)$ and it is significantly higher than preclinical subgroup $(P=0.014)$.

In this study, overall $68.3 \%$ of patients had increase RI values. Most of the patients belonging to preclinical subgroup $(70.5 \%)$ had normal RI value while most of the patients belonging to Incipient Nephropathy (72.7\%), overt Nephropathy (80\%) and Renal Failure subgroups $(90.9 \%)$ had increased RI values (Table1).

Table 1.Relative percentages of normal and high resistive indices (RI) value in various subgroups

\begin{tabular}{|l|l|l|l|}
\hline \multirow{2}{*}{ SUBGROUP } & \multicolumn{2}{|l|}{ RESISTIVE INDEX } & \multirow{2}{*}{ TOTAL } \\
\cline { 2 - 3 } & $\begin{array}{l}\text { NORMAL } \\
(<0.7)\end{array}$ & $\begin{array}{l}\text { HIGH } \\
(>0.7)\end{array}$ & \\
\hline PRECLINICAL & $12(70.5 \%)$ & $5(29.4 \%)$ & 17 \\
\hline $\begin{array}{l}\text { INCIPIENT } \\
\text { NEPHROPATHY }\end{array}$ & $3(27.2 \%)$ & $8(72.7 \%)$ & 11 \\
\hline $\begin{array}{l}\text { OVERT } \\
\text { NEPHROPATHY }\end{array}$ & $2(20 \%)$ & $8(80 \%)$ & 10 \\
\hline $\begin{array}{l}\text { RENAL } \\
\text { FAILURE }\end{array}$ & $2(9.1 \%)$ & $20(90.9 \%)$ & 22 \\
\hline TOTAL & 19 & 41 & 60 \\
\hline
\end{tabular}

The mean BUN level in preclinical, incipient nephropathy, overt nephropathy and renal failure subgroup were 18.47 $\pm 8.90 \mathrm{SD}$, 
18.18 $\pm 3.80 \mathrm{SD}, \quad 26.8 \pm 6.66 \mathrm{SD}, \quad 46.63 \pm 26.5 \mathrm{SD}$ respectively. In advanced DN, the mean BUN levels were higher and the variations in the BUN levels between the subgroups was found to be statistically significant $(P<0.001)$.

The mean serum creatinine level in preclinical, incipient nephropathy, overt nephropathy and renal failure subgroup were $0.93 \pm 0.25 \mathrm{SD}$, $0.89 \pm 0.16 \mathrm{SD}, \quad 1.18 \pm 0.16 \mathrm{SD}, \quad 3.6 \pm 2.00 \mathrm{SD}$ respectively.

Pearson correlation coefficients and linear regression analysis was used to assess correlation between Duplex ultrasound and biochemical parameters and results were presented as tabular form in table $2 \& 3$.

Table 2: Correlation between duplex ultrasound and biochemical parameters.

\begin{tabular}{|l|l|l|l|l|l|l|}
\hline \multirow{2}{*}{$\begin{array}{l}\text { BIOCHEM } \\
\text { ICAL }\end{array}$} & \multicolumn{6}{l}{ DUPLEX ULTRASOUND PARAMETERS } \\
\cline { 2 - 7 } $\begin{array}{l}\text { PARAMET } \\
\text { ERS }\end{array}$ & $\begin{array}{l}\mathrm{RK}_{\text {LENGT }} \\
\mathrm{H}\end{array}$ & $\begin{array}{l}\mathrm{LK}_{\mathrm{LE}} \\
\mathrm{NGTH}\end{array}$ & $\mathrm{PT}_{\mathrm{RK}}$ & $\mathrm{PT}_{\mathrm{LK}}$ & $\begin{array}{l}\text { ECHO } \\
\text { GRADE }\end{array}$ & $\begin{array}{l}\text { MEAN } \\
\text { RI }\end{array}$ \\
\hline FBS & 0.68 & 0.55 & 0.234 & 0.94 & 0.087 & 0.107 \\
\hline BUN & 0.46 & 0.198 & 0.24 & 0.193 & $<0.0001$ & $<0.0001$ \\
\hline $\begin{array}{l}\text { S.CREATI } \\
\text { NINE }\end{array}$ & 0.68 & 0.25 & 0.95 & 0.23 & 0.00013 & 0.00015 \\
\hline
\end{tabular}

(Note: The value in each cell represents " $r$ " value. A positive $r$ value depicts a positive correlation while a negative $r$ value represents negative correlation between the parameters. Very good positive correlation is represented by $r$ value of $>0.75$; RK- Right kidney, LKLeft kidney)
Table 3. Linear regression analysis between duplex ultrasound and biochemical parameters.

\begin{tabular}{|c|c|c|c|c|c|c|}
\hline \multirow{2}{*}{$\begin{array}{l}\text { BIOCHEMICAL } \\
\text { PARAMETERS }\end{array}$} & \multicolumn{6}{|c|}{ DUPLEX ULTRASOUND PARAMETERS } \\
\hline & $\begin{array}{l}\mathrm{RK}_{\mathrm{LE}} \\
\mathrm{NGTH}\end{array}$ & $\begin{array}{l}\mathrm{LK}_{\mathrm{L}} \\
\text { ENGT } \\
\mathrm{H}\end{array}$ & $\begin{array}{l}\mathrm{PT}_{\mathrm{R}} \\
\mathrm{K}\end{array}$ & $\mathrm{PT}_{\mathrm{LK}}$ & $\begin{array}{l}\text { ECHO } \\
\text { GRA } \\
\text { DE }\end{array}$ & $\begin{array}{l}\text { MEA } \\
\text { N RI }\end{array}$ \\
\hline FBS & $\begin{array}{l}- \\
0.054 \\
34\end{array}$ & $\begin{array}{l}0.07 \\
819\end{array}$ & $\begin{array}{l}- \\
0.15 \\
588\end{array}$ & -0.0093 & $\begin{array}{l}0.222 \\
53\end{array}$ & $\begin{array}{l}0.209 \\
69\end{array}$ \\
\hline BUN & $\begin{array}{l}0.006 \\
45\end{array}$ & $\begin{array}{l}- \\
0.05 \\
045\end{array}$ & $\begin{array}{l}- \\
0.15 \\
381\end{array}$ & - & $\begin{array}{l}0.536 \\
75\end{array}$ & $\begin{array}{l}0.506 \\
60\end{array}$ \\
\hline S.CREATININE & $\begin{array}{l}- \\
0.054 \\
15\end{array}$ & $\begin{array}{l}- \\
0.14 \\
82\end{array}$ & $\begin{array}{l}- \\
0.21 \\
732\end{array}$ & $\begin{array}{l}- \\
0.15678\end{array}$ & $\begin{array}{l}0.473 \\
15\end{array}$ & $\begin{array}{l}0.470 \\
28\end{array}$ \\
\hline
\end{tabular}

(Note: The value in each cell represents "p" value. Significance is suggested when the value is less than 0.05 ; RK- Right kidney, LKLeft kidney).

Blood urea nitrogen and serum creatinine showed a fair positive correlation with renal parenchymal echogenicity and resistive indices values. The results showed that with increase in Blood Urea Nitrogen and Serum creatinine level, there is a statistically significant increase (response) in renal parenchymal echogenicity grade and Resistive indices values.

\section{Discussion}

Duplex ultrasound study provides a reliable and non-invasive way of evaluating the kidney in Diabetic Nephropathy. Khati et al, ${ }^{[11]} 2005$, highlighted that Sonography is the best modality for evaluating the patients having renal insufficiency. Platt et al, ${ }^{[12]}$ Ishimura et al, ${ }^{[13]}$ Frauchiger et al ${ }^{[14]}$ and others also tried to study intrarenal vascular resistance in nephropathy patients.

Most of the patients in this study belonged to either renal failure subgroup $(36.67 \%)$ or in preclinical subgroup (28.33\%). This may be a result of referral of cases as ours is a tertiary care institute. With increase in mean duration 
of DM, there was also progressive increase in DN severity, which was found to be statistically significant $(P=0.02)$. Nejad et al ${ }^{[15]}$ reported the mean duration of Diabetes in end stage renal failure to be 15 years in his study. Patients were noted to end up in renal failure early (11.6 years) in this study when compared to other studies, this difference may have occurred due to different ethnicity and treatment regimen. Mohan et al ${ }^{[16]}$ reported the mean duration of Diabetes to be 9 years for development of microalbuminuria in Indian population and this study agrees with the same.

The renal length does not showed linear variation with increase in severity of $\mathrm{DN}$, but the largest kidneys were noted in preclinical subgroup. Mancini et al, ${ }^{[17]} 2013$, in his study reported that renal hypertrophy was present even in diabetic patients without proteinuria. There is progressive decrease in mean parenchymal thickness (PT) of kidneys were noted with increase in severity of DN, but it is not statistically significant. The renal failure subgroup had lowest PT. In a similar study done by Soldo et al ${ }^{[10]}$ with 3 subgroups of DN, advanced nephropathy showed lowest mean PT.

Out of 60 patients, only 14 (23.3\%) patients showed abnormal renal parenchymal echogenicity. In a similar study, $26 \%$ of total DN patient showed cortical hyperechogenicity and was seen only in advanced nephropathy. The echogenicity assessment is a subjective method but can be used to compare different patients if we do proper technique $\&$ use same depth for comparison ${ }^{[7]}$ and in our study we followed this correct technique. Siddappa et al, ${ }^{[8]} 2013$, said that the abnormal echogenicity suggest irreversible renal impairment changes.Hricak et $\mathrm{al}^{[7]}$ found that the parenchymal changes are due to affection of glomerulus, interstitium \& tubular structure and as such it is increased in various medical renal diseases and so it lacks accuracy in diagnosing diabetic renal disease.

The mean RI values in preclinical, incipient nephropathy, overt nephropathy and renal failure subgroup were $0.641 \pm 0.06 \mathrm{SD}$, $0.724 \pm 0.10 \mathrm{SD}, 0.756 \pm 0.05 \mathrm{SD}, 0.820 \pm 0.09 \mathrm{SD}$ respectively, showing progressive increase in mean RI values with increase in severity of DN, which was found to be statistically significant $(P<0.001)$. In preclinical stage, the mean RI value of $0.641 \pm 0.06 \mathrm{SD}$ is in normal range $(<0.70)$ and suggest inability of intra renal RI to detect changes in asymptomatic patients without microalbuminuria. However, in presence of microalbuminuria, the mean RI values are higher $(0.724 \pm 0.10)$ and it is significantly higher than preclinical subgroup $(P=0.014)$. Ishimura et al ${ }^{[13]}$ done study with similar subgroup and it shows mean RI in four subgroups as $0.698 \pm 0.058 \mathrm{SD}, 0.740 \pm 0.074 \mathrm{SD}$ $0.742 \pm 0.066 \mathrm{SD}, \quad 0.852 \pm 0.05 \mathrm{SD}$ respectively which was also statistically significant and the mean RI values were pathological for subgroups 2, $3 \&$ 4.Ishimura et al ${ }^{[13]}$ quoted Sauvian in support of his study and concluded that intrarenal RI values measurements were higher in microalbuminuric stage. Hamano et al, ${ }^{[18]} 2008$, also found that RI values were higher in diabetic patients with albuminuria than in patients without albuminuria.

In this study, overall $68.3 \%$ of patients had increase RI values. Most of the patients belonging to preclinical subgroup $(70.5 \%)$ had normal RI value while most of the patients belonging to Incipient Nephropathy (72.7\%), overt Nephropathy (80\%) and Renal Failure subgroups $(90.9 \%)$ had increased RI values indicating raised renal vascular resistance as the disease progresses. It might probably be indicating a raised peripheral vascular resistance due to arteriosclerosis in intrarenal vasculature. In the study done by Platt et al ${ }^{[12]}$ in diabetic patients where grouping is done such that the asymptomatic \& microalbuminuria patients were placed in 
same group, high RI was seen in 59\% of total type 2 DM patient studied and in $24 \%$ of patients without nephropathy, in $38 \%$ of patients with early nephropathy and in $96 \%$ of advanced nephropathy patients. Ghadirpour et $\mathrm{al}^{\left[{ }^{[19]}\right.} 2014$,also found in his study on DN that $90 \%$ of patients had higher RI values.

The mean BUN level in this study correlates with an earlier study done by Ishimura et al ${ }^{[13]}$ on similar subgroups of DN and the variations was found to be statistically significant $(P<0.001)$.

Pearson correlation coefficients and linear regression analysis was used to assess correlation between Duplex ultrasound and biochemical parameters.BUN showed fair positive correlation with renal parenchymal echogenicity $(\mathrm{r}=0.53 ; \quad P<0.001)$ and RI $(\mathrm{r}=0.50 ; P<0.001)$. Hricak et al ${ }^{[7]}$ had also showed significant correlation between BUN and renal parenchymal echogenicity but with wider range. Kawai et al, ${ }^{[20]} 2011$ also reported fair positive correlation between RI and BUN ( $\mathrm{r}=0.31 ; P<0.001)$ in their study on chronic kidney disease patients which included 70 diabetic patients. Serum creatinine showed fair positive correlation with renal parenchymal echogenicity $(\mathrm{r}=0.47 ; P<0.001)$ and RI $(\mathrm{r}=0.47 ; P<0.001)$. Platt et al ${ }^{[12]}$ had also showed similar strong positive correlation between serum creatinine and RI ( $r=0.64)$. Soldo et al ${ }^{[10]}$ had also showed similar strong positive correlation between serum creatinine and RI ( $r=0.711)$. Siddappa et al, ${ }^{[8]} 2013$, also showed significant correlation between serum creatinine and renal parenchymal echogenicity $(P=0.004)$.

\section{Limitations}

Our study was a cross sectional study and thus no follow up was possible and the changes in the various parameters over time could not be measured.

To determine and refine the role of this Doppler parameter in early microalbuminuric stage of DN, further prospective study with follow up is needed to obtain a better relationship with biochemical parameters \& progression of disease processes.

\section{Conclusion}

The present study demonstrates that the renal parenchymal echogenicity was noted to be increased only in advanced nephropathy. The Resistive Index (RI) was high in most of the Diabetic Nephropathy patients in 3 subgroups (incipient nephropathy, overt nephropathy and renal failure) and the mean RI was noted to be increasing with disease progression, which was statistically significant. This indicates its role as a Doppler parameter which can be used as complimentary to various biochemical parameters. The impact of diabetes mellitus and the duration of the disease on intra renal haemodynamics may be due to arteriosclerosis and interstitial lesions. Even though various ultrasound parameters were normal in nephropathy patients including renal parenchymal echogenicity, the RI values was noted to be raised and so the measurement of RI can add valuable information about vascular and interstitial components in diabetic renal disease. Intrarenal Doppler study can be used along with biochemical parameters in follow up of Diabetic Nephropathy and raised RI values should prompt the clinicians for aggressive management of such patients to delay the progression of diabetic renal disease. Our study was a cross sectional study and thus no follow up was possible and the changes in the various parameters over time could not be measured. To determine and refine the role of this Doppler parameter in early microalbuminuric stage of $\mathrm{DN}$, further prospective study with follow up is needed to obtain a better relationship with biochemical parameters \& progression of disease processes. 
Funding: None

Conflict of interest: None.

\section{References}

1. International Diabetes Federation. IDF Diabetes, 7 ed. Brussels, Belgium: International Diabetes Federation, 2015. http://www.diabetesatlas.org

2. Mehta SR, Kashyap AS, Das S. Diabetes Mellitus in India: The Modern Scourge.MJAFI 2009;65:50-4.

3. Joel F Platt. Duplex Doppler Evaluation of Native Kidney dysfuction: Obstruction and Nonobstructive disease. AJR 1992 May;158:1035-42.

4. Parolini C, Noce A, Staffolani E, Giarrizzo GF, Costanzi S, Splendiani $\mathrm{G}$. Renal resistive index and longterm outcome in chronic nephropathies. Radiology 2009;252:888-96.

5. Dissanayake VHW., Morgan L., Pipkin FB., Vathanan V., Premaratne S., Jayasekara RW, Seneviratne HR. The urine protein heat coagulation test-a useful screening test for proteinuria in pregnancy in developing countries: a method validation study. BJOG 2004;111:491-4. doi: 10.1111/j.14710528.2004.00128.x.

6. Toto RD. Microalbuminuria: Definition, Detection and Clinical Significance. J ClinHypertens (Greenwich). 2004 nov;6(11):2-7.

7. Hricak H, Cruz C, Romanski R, Uniewski MH, Levin NW, Madrazo BL,et al. Renal Parenchymal Disease : Sonographic-Histologic Correlation. Radiology 1982 July;144:141-7.

8. Siddappa JK, Singla S, Al Ameen M, Rakshith S C, Kumar N. Correlation of Ultrasonographic Parameters with Serum Creatinine in Chronic Kidney Disease. J ClinImaging Sci 2013;3:28. doi: 10.4103/2156-7514.114809.
9. Pourcelot L. Applications cliniques de l'examen Doppler Transcutane. In: Peronneau $\mathrm{P}$ ed. Velocimetrie Ultrasonore Doppler INSERM, Paris 1975;213-40.

10. Soldo D, Brkljacic B, Bozikov V, Drinkovic I, Hauser M. Diabetic nephropathy. Acta Radiologica 1997;38 (2):296-302.

11. Khati NJ, Hill MC, Kimmel PL. The role of ultrasound in renal insufficiency: the essentials Ultrasound Q 2005 Dec;21(4):227-44.

12. Platt JF, Rubin JM, Ellis JH. Diabetic Nephropathy: Evaluation with Renal Duplex Doppler US. Radiology 1994;190:343-6.

13. Ishimura E, NishizawaY, Kawagishi T, Okuno Y, Kogawa K, FukumotoS, et al. Intrarenal hemodynamic abnormalities in Diabetic nephropathy measured by duplex Doppler sonography. Kidney International 1997;51:1920-7.

14. Frauchiger B, Nussbaumer P, Hugentobler M, Staub D. Duplex sonographic registration of age and diabetes-related loss of renal vasodilatorry response to nitroglycerine. Nephrol Dial Transplant 2000;15:827-32

15. Nejad MN, Jafari B, Alipour P. Arterial Resistive Index (RI) in Type II Diabetic Nephropathy Stages and Healthy Controls. Iran $\mathbf{J}$ Radiol 2009;6(1):29-32.

16. Mohan MM, V CS, Prevalence and risk factors of microalbuminuria in type 2 diabetes mellitus. Int J Adv Med 2015;2(4):383-86. doi:10.18203/23493933.ijam20151014.

17. Mancini M, Masulli M, Liuzzi R, Mainenti RP, Ragucci M, MaureaS,et al.Renal Duplex Sonographic Evaluation of Type 2 Diabetic Patients. 
J Ultrasound Med2013 June

1;32(6):1033-40.doi:

10.7863/ultra.32.6.1033.

18. Hamano K, Nitta A, Ohtake T, Kobayashi S. Associations of Renal Vascular Resistance With Albuminuria and Other Macroangiopathy in Type 2 Diabetic Patients. Diabetes Care 2008 Sept;31(9);1853-7. doi: 10.2337/dc080168.

19. Ghadirpour A, Tarzamni MK, Naghavi-Behzad M, Abedi-Azar S, KoushavarH,Nezami N. Med Ultrason 2014;16(2):95-9. doi: 10.11152/mu.2013.2066.162.ag1mkt2.

20. Kawai T, Kamide K, Onishi M, Yamamoto-Hanasaki H, Baba Y, HongyoK,et al. Usefulness of the resistive index in renal Doppler ultrasonography as an indicator of vascular damage in patients with risks of atherosclerosis. Nephrol Dial Transplant 2011;26: 3256-62. doi: 10.1093/ndt/gfr054. 